\title{
Distributed Photonic Instrumentation for Smart Grids
}

\author{
Philip Orr, Campbell Booth, Grzegorz Fusiek, \\ Pawel Niewczas and Adam Dysko \\ Institute for Energy and Environment \\ University of Strathclyde \\ Glasgow, United Kingdom \\ philip.orr@strath.ac.uk
}

\author{
Fumio Kawano and Phil Beaumont ${ }^{\mathrm{b}}$ \\ ${ }^{\mathrm{a}}$ Toshiba Corporation, Tokyo, Japan \\ ${ }^{\mathrm{b}}$ Toshiba International (Europe), London, United Kingdom
}

\begin{abstract}
Photonic sensor networks possess the unique potential to provide the instrumentation infrastructure required in future smart grids by simultaneously addressing the issues of metrology and communications. In contrast to established optical CT/VT technology, recent developments at the University of Strathclyde in distributed point sensors for electrical and mechanical parameters demonstrate an enormous potential for realizing novel and effective monitoring and protection strategies for intelligent electrical networks and systems. In this paper, we review this technology and its capabilities, and describe recent work in power system monitoring and protection using hybrid electro-optical sensors. We show that wide-area visibility of multiple electrical and mechanical parameters from a single central location may be achieved using this technology, and discuss the implications for smart grid instrumentation.
\end{abstract}

Keywords-instrumentation, sensors, protection, photonics, optics, smart grids

\section{INTRODUCTION}

Since their inception almost 40 years ago, optical fiber sensors have evolved - driven principally by research in the academic and military sectors - to become a benchmark in high-performance instrumentation [1,2]. The robustness, flexibility, geometry, and performance of fiber sensors are such that for many measurement applications it is the only viable technology. In particular, the ability of fiber sensor systems to serially-multiplex high numbers of sensors for a wide range of mechanical and electromagnetic measurands lends them to the efficient instrumentation of a multiplicity of power system networks and components. The use of such extensive optical sensor networks in other highly-demanding applications (e.g. military subsea hydrophone arrays, structural health monitoring schemes, or perimeter alarms) has now been well established $[3,4]$, and it is apt that the unique benefits of optical fiber sensors are now considered seriously for use more broadly in power system instrumentation - particularly in light of the present drive towards higher levels of visibility of voltages, current, and mechanical parameters at numerous and diverse locations within power systems [5].

Commercial optical current transducers (OCTs) are now relatively well established and are developed by a range of manufacturers for their passive and highly accurate measurement of electrical current [6,7]. The common design is based on either spun or annealed optical fiber (to reduce shapeinduced linear birefringence) that is coiled around the conductor or current path. The contour integral of the circulating magnetic field around the conductor then yields a polarimetric or interferometric measurement of enclosed current based on the Faraday effect. Optical voltage transducers (OVTs) have also been developed, primarily based upon either the electro-optic (Pockels) effect or the piezoelectric effect [8]. Although OCT and OVT technologies have experienced notable commercial success, both rely on interferometric or polarimetric measurement techniques, limiting the distance from interrogator to measurement point to under $\sim 100$ metres. Additionally, similarly to conventional electrical transducers, it is not possible to discriminate between superimposed sensor responses and thus serial multiplexing is not possible. For these reasons, wide-area coverage, measurement over long distances, and high numbers of sensors are not presently achieved by commercially-available optical current or voltage measurement schemes.

In contrast to the existing OCT/OVT technology, we present in this article a quasi-distributed sensing methodology for voltage, current, and mechanical parameters. We firstly describe the transducer and measurement system, and discuss the associated capability for distributed, passive measurements. We then describe recent developments and demonstrations of distributed optical sensing in power systems, and present a vision for the effective and thorough instrumentation of smart grids using this technology. The article is intended to generate discussion regarding the future of optical sensor networks for facilitating successful smart grids.

\section{POINT TRANSDUCERS FOR VOLTAGE AND CURRENT}

Transducers for electrical current may be classified as: (i) conventional, i.e. utilizing resistive shunts or iron core or Rogowski type transformers; (ii) pure optical based on the Faraday effect as described in Section I; or (iii) extrinsic optical. It is sensors of the latter category that are the focus of this paper, where an intermediate transducer is used to convert current into a secondary quantity - strain in this instance - that is measurable by the optical transducer.

\section{A. Fiber Bragg gratings (FBGs)}

Fiber Bragg gratings (FBGs) are periodic perturbations of the refractive index along a fiber core, having peak optical reflection at a specific wavelength, known as the Bragg wavelength [9] and a typical physical length of 5-10 $\mathrm{mm}$. In 
sensor applications, their wavelength-encoding nature, coupled with their simple reflected spectra, means that FBGs are relatively easy to interrogate and multiplex, and are effectively immune to the problems of intensity fluctuations and attenuation [1]. For these reasons the FBG is now ubiquitous in the field of optical instrumentation [10].

Peak optical reflection from FBGs occurs at a wavelength $\lambda$ equal to twice the grating period, i.e. at $\lambda / n=2 \Lambda$ where $\mathrm{n}$ is the fiber refractive index and $\Lambda$ is the pitch of the grating. Thus, straining or compressing the fiber longitudinally at the location of the grating shifts up or down, respectively, the peak reflected wavelength. Illumination of the FBG by broadband light, and some form of peak wavelength detection and tracking, may therefore be employed to utilize the FBG as a strain sensor.

\section{B. FBG-based voltage and current sensors}

The authors have developed fiber-optic voltage and current point transducers, based on FBG technology, that have been applied successfully to power system plant diagnostics $[11,12]$. The complete optical sensor system has been shown to be capable of measuring dynamically changing signals and has been successfully used for detecting higher order voltage and current harmonics [13]. The hybrid voltage sensor utilizes an FBG bonded to a multilayer piezoelectric stack, while the current sensor uses a small, high-bandwidth current transformer monitored by a dedicated voltage sensor as shown in Fig. 1. In both cases an FBG peak wavelength shift can be calibrated in terms of voltage or current, and a temperature measurement can also be performed simultaneously using the same sensors.
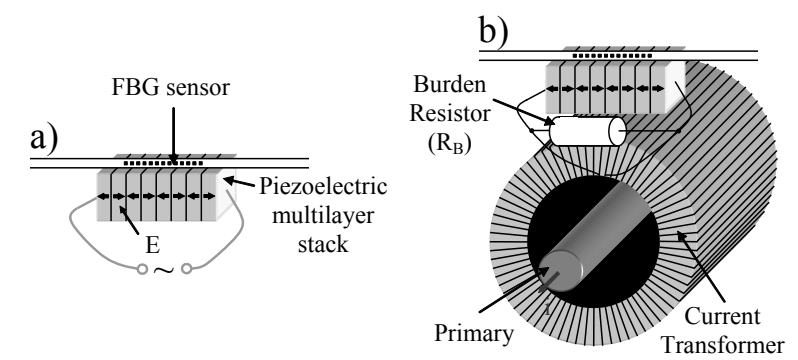

Fig. 1. (a) Hybrid piezoelectric voltage sensor. (b) Hybrid current sensor employing a voltage sensor and a current transformer.

It was demonstrated previously that these fiber-optic voltage and current sensors can be used for measuring variable frequency voltage and current waveforms for use in future aero-electric power systems [14]. Their use in simplified bespoke differential and distance protection schemes (implemented within the sensor interrogation unit) has also been reported in a preliminary form [15] without integration with protection hardware. Regarding the accuracy of the voltage sensor, uncertainty in the measurement will be introduced primarily by imperfect calibration of the strain/volt coefficient attributed to the piezoelectric multilayer stack however this source of error can be minimized by proper stack characterization. Other more minor error contributions can result from imperfect knowledge of the nominal wavelength of the FBG, and imperfect strain transfer from the stack to the optical fiber via the chosen bonding mechanism.

\section{SENSOR NETWORKING AND INTERROGATION}

In the generic architecture of an FBG sensor interrogation scheme, light from an optical source is guided by fiber to an array of serially-multiplexed FBGs. Reflections from all FBGs are returned via a coupler to the interrogating device, at which the peak reflected wavelength from each sensor is extracted.

While a multitude of sensor interrogation techniques exist, the measurement resolution provided by optical interferometry is unmatched. For this reason, we have recently developed FBG interrogation and multiplexing technology based upon Mach-Zehnder interferometry that improves upon previous techniques $[16,17]$ in order to deliver absolute, static, and dynamic measurements of FBG reflected wavelengths [18]. This combination of measurement attributes is critical for many power and energy instrumentation applications (e.g. dc voltage, pressure, static strain, temperature). Fig. 2 illustrates this preferred technique.

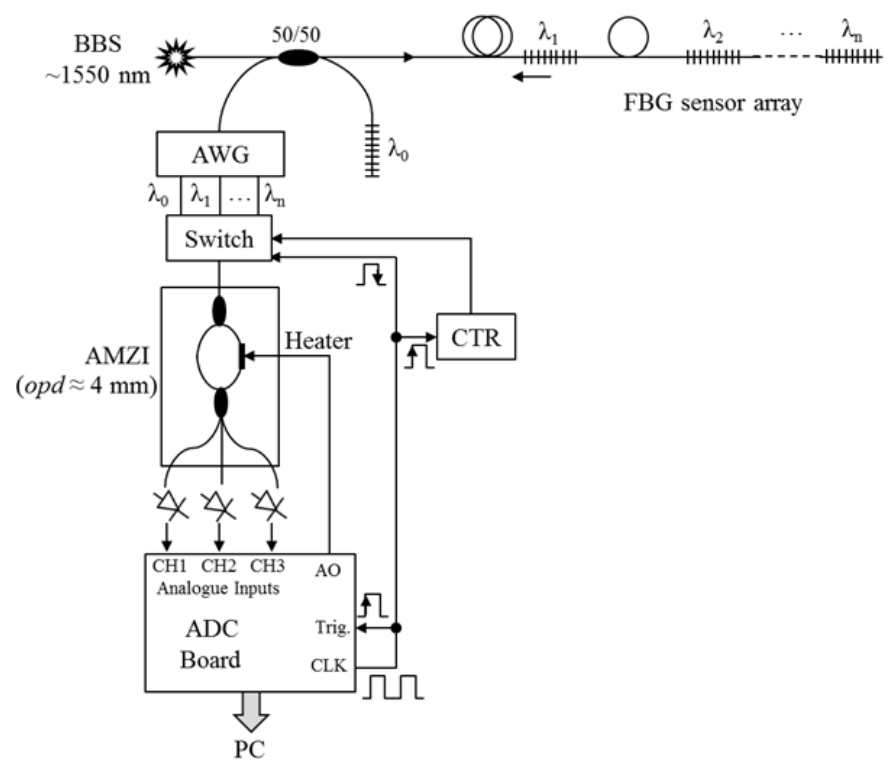

Fig. 2. General architecture of a fast solid-state interrogation system for measurement of quasistatic or dynamic shifts in FBG sensor wavelengths. BBS is broadband source, WDM is passive wavelength division multiplexer, UMZI is unbalanced Mach-Zehnder interferometer.

While further detail on the specifications and architecture of this interrogation system can be found in [18], we have demonstrated in practice its capability to acquire signals from a multitude of FBG sensors (configured for measurement of voltage, current, vibration, or temperature) down to a strain measurement resolution of $10 \mathrm{n} \varepsilon / \sqrt{ } \mathrm{Hz}$ - corresponding to a voltage measurement resolution of $0.6 \mathrm{mV} / \sqrt{\mathrm{Hz}}$ using commercially available piezoelectric stacks at a measurement distance of $20 \mathrm{~km}$. Thus, the combination of the proposed transducers and interrogation scheme present the possibility for high-resolution passive measurement of voltages and currents at many diverse locations on a power network.

The cost of FBG sensor multiplexers or interrogators scales, in general, with both the measurement acquisition rate and the size of the FBG sensor array. While interrogators may be priced on a par with commercial protection relays, there is a 
cost reduction benefit associated with the adoption of this technology for multiple measurements at multiple locations on the network, since unlike standard technologies there is no requirement for duplication of the measurement system or location of the measurement system near sensing points. It is also important to note the substantial drop in FBG sensor costs over the past decade, resulting in a number of cheap, highperformance transducers compatible with this approach. Overall, therefore, the capability for wide-area coverage of multiple measurands will enable a reduction in costs overall due to the technology's extensibility and centralized nature.

\section{COMPATIBILITY WITH EXISTING SYSTEMS}

While bespoke monitoring systems based upon the described technology may be developed, it is also possible and desirable to deliver optically-acquired measurements to existing systems to exploit established monitoring or protection techniques. The authors have demonstrated such compatibility with the commercially-available Toshiba's GRB100 busbar protection relay [19]. Fig. 3. illustrates the electrical scenario used for delivery of internal and external faults to a three-ended test network.

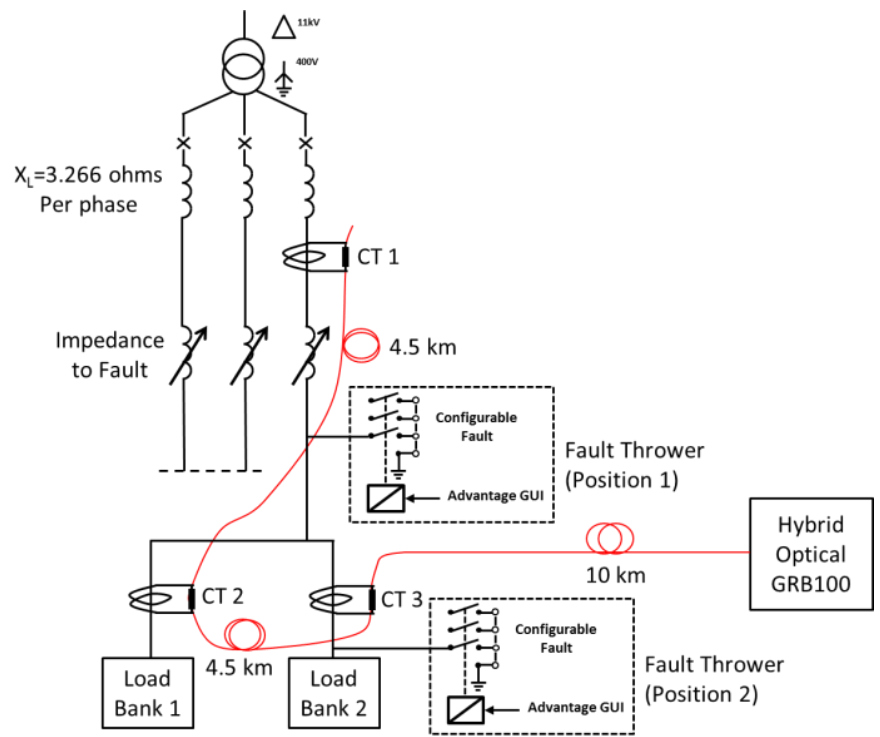

Fig. 3. Three-ended laboratory network used to demonstrate passive optical acquisition of currents for unit protection in tandem with a GRB100 protection relay. CTs are interrogated passively by a single optical fiber of total length 19 $\mathrm{km}$.

Fig. 4 illustrates a single case of internal phase-earth fault applied to the network in Fig. 3. In this figure, (a) shows the signals as stored by the GRB100 fault recorder after correct tripping.

In these tests, the output of the sensor interrogation scheme was configured to emulate the bespoke communication protocol of Toshiba's GRB100 bay units. This allowed the optical sensor technology to be retrofitted to an un-modified GRB100 system in order to deliver multiple widely-spaced current measurements to the unit in the format expected from three individual bay units. This represents an important demonstration of the improved flexibility and functionality that could be achieved by commercial optical measurement systems designed to pass centrally-acquired voltage and current values to existing well-established relaying systems in bespoke or universally-accessible formats such as IEC-61850 [20].
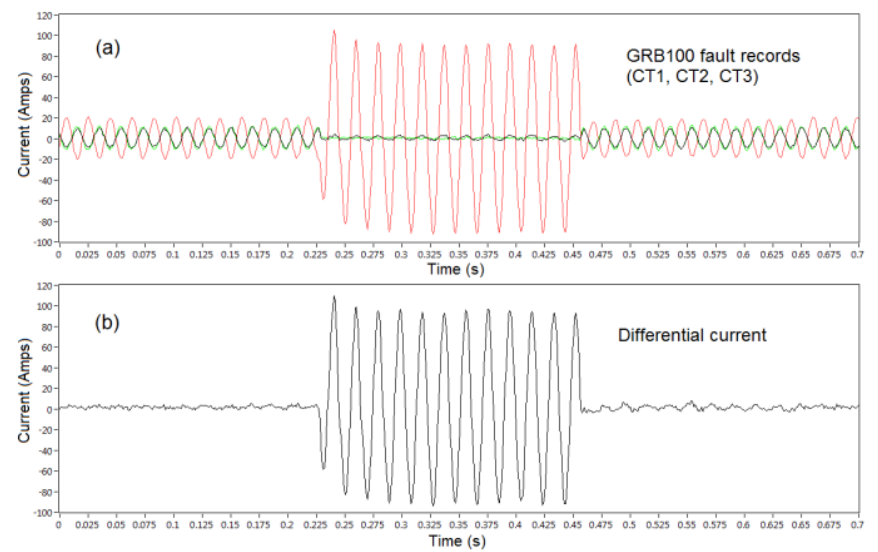

Fig. 4. (a) Internal fault (65A fault current) as recorded simultaneously by three passive optical current sensors on a single fiber (CT1, CT2, CT3 from Fig. 3). Each sensor was separated physically by $4.5 \mathrm{~km}$. (b) The differential current.

Further detail on these particular tests may be found in [21], however the general principal of compatibility between passive, multiplexed optical measurements and existing protection and monitoring hardware is clearly illustrated.

\section{NEW SYSTEMS AND APPROACHES}

A number of specific benefits to power system instrumentation may be indicated based upon the technology outlined in this paper:

- minimization of communication infrastructure physically small transducers and fibers, the use of multiplexed sensors, and the combination of electrical and mechanical transducers allow the sensing system to be efficient and compact while delivering a range of high-resolution measurements;

- minimization of local measurement units and associated power sources - unlike conventional relaying systems, independent measurement units near measurement locations are not required, nor are associated lowvoltage de supplies;

- improved speed of operation owing to minimization of ADC/DAC and digital communication bottlenecks using a centralized architecture, ADC operations are performed only once in the data flow, while the lack of digital communications removes bandwidth issues that will become increasingly problematic as sensor numbers increase to meet Smart Grid specifications;

- flexibility in measurement location owing to the use of passive transducers addressed purely by telecomsgrade optical fiber - measurement over distances as long as $100 \mathrm{~km}$ are possible using fiber-optic sensors, and sensor fibers are entirely compatible with telecommunication fibers that may already be installed along key data routes; 
- the potential for entirely new protection algorithms the visibility of multiple voltages and currents by a single central system will allow centralized protection algorithms - often restricted to deployment in power systems with a high level of local telecommunication infrastructure (e.g. shipboard systems) - to be deployed more generally and in situations not previously tenable.

These benefits give rise to key applications of the technology in developing new and bespoke monitoring and protection schemes - often where conventional technology cannot meet demands. While many such applications exist, we list a few here by way of illustration:

\section{A. Protection of hybrid overhead/underground lines}

In present protection schemes, it is not possible to discriminate which section of a hybrid line is subject to the fault, and to install additional measurement and protection devices at the cable terminals is costly and can be impractical. An optical fiber multi-differential relay could be developed that would provide passive current measurement at multiple locations along the hybrid line, including at cable terminals. In this way, the relay would be able to determine, with a high level of discrimination, which section of the line is faulted and could initiate (or inhibit) re-closure accordingly.

\section{B. Fast and simpler multi-terminal protection}

Compared to the centralized, passive optical approach, present multi-terminal protection architecture is expensive and convoluted, requiring many relays, dedicated communication channels, and sampling synchronization control. Building upon the preliminary demonstration in Section IV, it will be possible to increase the breadth of coverage in terms of sensor numbers and coverage area, thus facilitating the development of a centralized protection scheme that can operate over wide areas with no dedicated communications infrastructure.

\section{UHV transformer protection}

Protection and monitoring of UHV transformers is extensive and complex due to the requirement for multiple measurement points [22]. Since FBG sensors are capable of measuring electrical parameter $(\mathrm{V}, \mathrm{I})$ and others such as temperature, vibration, and pressure, a single "protection and monitoring" scheme could therefore be developed on this basis, delivering full electrical and mechanical coverage of these large, valuable items of plant.

\section{Fiber-enabled synchrophasors (without GPS)}

Presently, phasor measurement units (PMUs) require use of the GPS network to accurately time-stamp voltage or current measurements for direct comparison of phasors at diverse locations on the network [23]. With an optical scheme, the relative delay between sensing points is measurable. A constant delay of approximately $4.9 \mu \mathrm{s} / \mathrm{km}$ will exist between sensor reflections arriving at the interrogation unit, which can be corrected for at the central interrogator/relay. Since FBG interrogators can make measurements across a span of $100 \mathrm{~km}$ or more, there is potential for centralized wide-area synchrophasor coverage without a requirement for absolute time-stamping or access to the GPS network.

\section{CONCLUSIONS}

In this paper, we have reviewed the methodology and capabilities of quasi-distributed fiber-optic instrumentation for voltage, current, and a range of other compatible mechanical parameters. The key benefit of this technology compared with commercial optical or conventional instrumentation is its capability for wide-area and passive interrogation of electrical and mechanical parameters from a single central location. We have briefly introduced a high-performance interrogation architecture for such sensors that allows the retention of interferometric measurement resolution (equating to a voltage measurement noise floor of $0.6 \mathrm{mV} / \sqrt{\mathrm{Hz}}$ ) while facilitating absolute, static, and dynamic measurement of FBG wavelengths, and demonstrated the compatibility of the optical instrumentation with existing commercial protection relaying hardware (GRB100 relay manufactured by Toshiba). Finally, we have discussed the excellent potential for such technology to facilitate novel or improved functionality in a range of power system protection and monitoring scenarios.

\section{ACKNOWLEDGMENT}

The authors acknowledge the funding and collaboration of Toshiba International (Europe) and Toshiba Corporation.

\section{REFERENCES}

[1] B. Lee, "Review of the present status of optical fiber sensors," Optical Fiber Technology, vol. 9, pp.57-79, 2003.

[2] Y. J. Rao, “ In-fiber Bragg grating sensors,” Measurement Science and Technology, vol. 8, pp.355-375, 1997.

[3] G.A.Cranch, P.J.Nash, "Large-scale arrays of fiber-optic interferometric sensors using TDM and DWDM" IEEE J. Light.Tech., vol. 19, no. 5, pp. 697-699, 2001

[4] M. Majumder, T. K. Gangopadhyay, A. K. Chakraborty, K. Dasgupta, D.K. Bhattacharya, "Fiber Bragg gratings in structural health monitoring-Present status and applications," Sensors and Actuators A: Physical, vol. 147, no. 1, pp.150-164, 2008.

[5] U.S. Department of Energy, "The smart grid: An introduction," Washington, DC, 2008.

[6] Alstom NXT Phase COSI Products, http://www.nxtphase.com (Accessed 29/05/2013)

[7] K. Bonhert, P. Gabus, J. Kostovic, H. Brandle, "Optical fiber sensors for the electric power industry," Optics and Lasers in Engineering, vol. 43, pp. 511-526, 2005

[8] Pan, F., Xiao, X., Xu, Y., and Ren, S., "An Optical AC Voltage Sensor Based on the Transverse Pockels Effect," Sensors (Basel), vol. 11, no. 7, pp.6593-6602, 2011.

[9] G. Meltz, W. W. Morey, W. H. Glenn, "Formation of Bragg gratings in optical fibers by a transverse holographic method," Opt. Let., vol. 14, no. 15, pp. 823-825, August 1989.

[10] A. Mendez, "Fiber Bragg grating sensors: a market overview," Proc. SPIE, vol. 6619, p. 661905, 2007.

[11] L. Dziuda, P. Niewczas, G. Fusiek, J. R. McDonald, "Hybrid FiberOptic Voltage Sensor for Remote Monitoring of Electrical submersible Pump Motors", Optical Engineering, Vol. 44, No. 6, pp 64401-1-6, June 2005

[12] L. Dziuda, G. Fusiek, P. Niewczas, G. Burt, and J.R. McDonald, "Laboratory Evaluation of the Hybrid Fiber-Optic Current Sensor", 
Sensors and Actuators, A: Physical, Vol. 136, No. 1, pp. 184-190, May 1,2007

[13] P. Niewczas, G. Fusiek, J. R. McDonald, "Dynamic capabilities of the hybrid fiber-optic voltage and current sensors", IEEE Sensors Conference, Daegu, Korea, Oct. 22-25, 2006

[14] G. Fusiek, P. Niewczas, J. R. McDonald, "Concept Level Evaluation of the Optical Voltage and Current Sensors and an Arrayed Waveguide Grating for Aero-Electrical Systems' Applications", the 24th IEEE IMTC 2007 Instrumentation and Measurement Technology Conference, Warsaw, Poland

[15] P. Orr, P. Niewczas, A. Dysko, C. Booth, F. Kawano, G. Baber, "Distributed optical distance protection using FBG-based voltage and current transducers," IEEE Power and Energy Society General Meeting, pp. 1-5, 24-29 July 2011

[16] A. D. Kersey, T. A. Berkoff, and W. M. Morey, "Multiplexed fiber Bragg grating strain-sensor system with a fiber Fabry-Perot wavelength filter," Opt. Lett., vol. 18, pp. 1370-1372, 1993.

[17] T. A. Berkoff and A. D. Kersey, "Fiber Bragg grating array sensor system using a bandpass wavelength division multiplexer and interferometric detection," Phot. Tech. Lett., vol. 8, no. 11, pp. 1522$1524,1996$.
[18] P. Orr and P. Niewczas, "High-Speed, Solid State, Interferometric Interrogator and Multiplexer for Fiber Bragg Grating Sensors," IEEE J. Lightwave Tech., vol. 29, no. 22, pp.3387-3392, 2011.

[19] GRB100 Busbar Protection, http://toshiba.co.jp/sis/en/tands/protect/grb100.htm, Toshiba Corporation (Accessed 29/05/2013)

[20] R. Mackiewicz, "Overview of IEC 61850 and Benefits," in Transmission and Distribution Conference and Exhibition, 2005/2006 IEEE PES, 2006, pp. 376-383

[21] P. Orr, G. Fusiek, P. Niewczas, C.D. Booth, A. Dysko, F. Kawano, T. Nishida, P. Beaumont, "Multi-point and centralized protection schemes using distributed fiber-optic sensors," IEEE. Trans. Power Delivery (under review)

[22] X. Tang, K. Kobayashi, Y. Sonobe, M. Okazaki, A. Morimoto, P. Beaumont, "Development of $765 \mathrm{kV}$ Transformer Protection Relay," Proc. Advanced Power System Automation and Protection, 2011.

[23] I. Hall, P. G. Beaumont, G. P. Baber, I. Shuto, M. Saga, K. Okuno, H. Ito, "New line current differential relay using GPS sycnhronization," Proc. IEEE Power Tech Conference, Bologna, p. 8, June 2003 